\title{
Palindromic polynomials, time-reversible systems, and conserved quantities
}

\author{
Ivan Markovsky and Shodhan Rao
}

\begin{abstract}
The roots of palindromic and antipalindromic polynomials appear in pairs $(\lambda, 1 / \lambda)$. A polynomial with such roots is antipalindromic if and only if in addition, it has a root at 1 of an odd multiplicity. The result has applications in system theory: 1) any kernel representation of a discrete-time, timereversible, scalar, autonomous LTI system is either palindromic or antipalindromic. (Similar statement holds for systems with inputs.) 2) LTI systems with palindromic or antipalindromic kernel representations have nontrivial conserved quantities.
\end{abstract}

\section{INTRODUCTION}

Links between roots patterns and coefficients patterns of polynomials have been extensively studied in the context of dynamical systems and control. A famous result of this type is the Routh-Hurwitz stability test, which allows to check the stability of a single input single output (SISO) linear timeinvariant system without computing its poles, i.e., by a finite number of operations on the coefficients of a differential equation representation of the system.

We study the root location of palindromic and antipalindromic polynomials, i.e., polynomials whose coefficients are respectively symmetric and antisymmetric with respect to the middle coefficient. It turns out that an autonomous discrete-time linear time-invariant (LTI) system defined by a difference equation whose coefficients are palindromic or antipalindromic is time-reversible in the sense that any trajectory of that system reversed in time is also a trajectory of the system. The continuous-time analogue of the palindromic and antipalindromic polynomials are even and odd polynomials.

Time-reversible systems have been studied in [FW91]. In this paper, we give more details (Theorems 10 and 11) about the structure of scalar autonomous and SISO time-reversible systems. Furthermore, we show that time-reversible systems possess conserved quantities. These are quadratic functionals of the system variables that remain constant in time along any trajectory of the system.

For both palindromic and antipalindromic polynomials a root $\lambda$ has a corresponding root $1 / \lambda$ of the same multiplicity. The property that distinguishes palindromic from antipalindromic polynomials is the multiplicity of the root at 1 . In the case of simple roots, a palindromic polynomial has no root at 1 , while an antipalindromic polynomial has a root at 1 .

We assume that the reader is familiar with the concepts of behavioral system theory and the calculus of quadratic dif-

The authors are with the School of Electronics and Computer Science, University of Southampton, Southampton, SO17 1BJ, UK imdecs.soton.ac.uk, sr05rdecs.soton.ac.uk ferential forms (QDFs) and we refer respectively to [PW97] and [WT98] for thorough exposition of these concepts.

\section{PALINDROMIC AND ANTIPALINDROMIC POLYNOMIALS}

In this section, we study the root location of palindromic and antipalindromic polynomials.

Definition 1 (Palindromic and antipalindromic polynomials). A polynomial $p \in \mathbb{R}[\xi]$ of degree $n$

$$
p(\xi)=p_{0}+p_{1} \xi+\cdots+p_{n} \xi^{n}, \quad p_{n} \neq 0
$$

is palindromic if its coefficients $p_{0}, p_{1}, \ldots, p_{n}$ form a palindrome, i.e.,

$$
p_{i}=p_{n-i}, \quad \text { for } i=0,1, \ldots, n .
$$

A polynomial $p$ is antipalindromic if $p_{i}=-p_{n-i}$, for $i=$ $0,1, \ldots, n$.

The following theorem is the main result of this section.

Theorem 2 (Root location of palindromic and antipalindromic polynomials). The polynomial $p \in \mathbb{R}[\xi]$ is palindromic/antipalindromic if and only if

1) every root $\lambda \in \mathbb{C}$ of $p$ is either on the unit circle or $p$ has a root $1 / \lambda$ with the same multiplicity as $\lambda$, and

2) $1+\mathbf{i} 0$ is a root of $p$ of even/odd multiplicity (multiplicity 0 means that $1+\mathbf{i} 0$ is not a root of $p)$.

In addition, if $p$ has an odd degree, then $-1+\mathbf{i} 0$ is a root of $p$ of an odd/even multiplicity.

Proof: First, note that item 1 in the statement of Theorem 2 is equivalent to:

1') For every root $\lambda \in \mathbb{C}$ of $p$ there exists a root $1 / \lambda$ with the same multiplicity as $\lambda$.

Indeed, if $\lambda$ is complex and is on the unit circle, then $1 / \lambda=\bar{\lambda}$ and by the assumption that $p$ has real coefficients, it follows that $1 / \lambda$ is also a root of $p$ with the same multiplicity. If $\lambda= \pm 1$, then $1 / \lambda=\lambda$ and (1') is trivially satisfied.

Let $\lambda_{1}, \ldots, \lambda_{k}$ be the distinct roots of $p$ and let $n_{1}, \ldots, n_{k}$ be their respective multiplicities. Note that the assumptions $p_{n} \neq$ 0 and $p$ palindromic or antipalindromic imply that $\lambda_{i} \neq 0$ for all $i$. Denote the $j$ th derivative of $p(\xi)$, evaluated at $\lambda \in \mathbb{C}$ by $\left.\frac{\mathrm{d}^{j}}{\mathrm{~d} \xi^{j}} p(\xi)\right|_{\xi=\lambda}$. A new characterisation of palindromic and antipalindromic polynomials, can be written as

\footnotetext{
$p$ is palindromic/antipalindromic

with distinct roots $\lambda_{1}, \ldots, \lambda_{k}$

of respective multiplicities $n_{1}, \ldots, n_{k}$
} 


$$
\left\{\begin{array}{l}
\left.\frac{\mathrm{d}^{j}}{\mathrm{~d} \xi^{j}}\left(p(\xi) \mp \xi^{n} p(1 / \xi)\right)\right|_{\xi=\lambda_{i}}=0, \text { for } i=1, \ldots, k, \\
j=0, \ldots, n_{i}-1, \text { and } p_{0} \mp p_{n}=0 .
\end{array}\right.
$$

For each $i \in\{1, \ldots, k\}$, the first condition gives the system of equations $A\left(\lambda_{i}\right) P\left(\frac{1}{\lambda_{i}}\right)=0$, where

$$
\begin{aligned}
& A(\lambda)=\left[\begin{array}{ccccc}
\lambda^{n} & 0 & \cdots & \cdots & 0 \\
n \lambda^{n-1} & -\lambda^{n-2} & 0 & \cdots & 0 \\
n(n-1) \lambda^{n-2} & -2(n-1) \lambda^{n-3} & \lambda^{n-4} & \ddots & \vdots \\
\vdots & \vdots & \vdots & \vdots & 0 \\
* & * & * & * & \lambda^{0}
\end{array}\right] \\
& P(\xi)=\operatorname{col}\left(p(\xi), \frac{\mathrm{d}}{\mathrm{d} \xi} p(\xi), \ldots, \frac{\mathrm{d}^{n_{i}-1}}{\mathrm{~d} \xi^{n_{i}-1}} p(\xi)\right)=0 .
\end{aligned}
$$

The coefficient matrix in the left hand side is lower-triangular with nonzero diagonal elements (since $\lambda_{i} \neq 0$ ). Therefore,

$$
p\left(1 / \lambda_{i}\right)=\frac{\mathrm{d}}{\mathrm{d} \lambda} p\left(1 / \lambda_{i}\right)=\cdots=\frac{\mathrm{d}^{n_{i}-1}}{\mathrm{~d} \lambda^{n_{i}-1}} p\left(1 / \lambda_{i}\right)=0,
$$

which proves that $1 / \lambda_{i}$ is a root of $p$ with multiplicity $n_{i}$. We showed that

$$
\begin{gathered}
p \text { is palindromic/antipalindromic } \Longleftrightarrow \\
\left\{\text { item 1' holds and } p_{0} \mp p_{n}=0,\right.
\end{gathered}
$$

i.e., any polynomial satisfying item 1 ' is either palindromic or antipalindromic and, vice verse, any palindromic or antipalindromic polynomial satisfies item 1'. The condition $p_{0} \mp p_{n}=0$ distinguishes between the palindromic and the antipalindromic case. In order to complete the proof, we have to show that

item 1' holds and $p_{0} \mp p_{n}=0 \Longleftrightarrow$ items 1 ' and 2 hold.

This equivalence follows from recursive application of the following lemmas.

Lemma 3. The polynomial $p \in \mathbb{R}[\xi]$ is antipalindromic if and only if there is a palindromic polynomial q, such that

$$
p(\xi)=(\xi-1) q(\xi) .
$$

Lemma 4. The polynomial $p \in \mathbb{R}[\xi]$ is palindromic and has a root at +1 if and only if there is an antipalindromic polynomial q, such that (1) holds.

Let $p$ be palindromic. If $p$ has no root at +1 , we are done (the multiplicity of +1 is 0 ). If $p$ has a root at +1 , then, according to Lemma 4 , it can be factored into $(\xi-1) p^{(1)}$, where $p^{(1)}(\xi)$ is antipalindromic. According to Lemma $3, p^{(1)}$ can be factored as $(\xi-1) p^{(2)}(\xi)$, were $p^{(2)}$ is palindromic. At this stage, $p=(\xi-1)^{2} p^{(2)}$ and we repeat the argument above replacing $p$ with $p^{(2)}$. In general, the procedure terminates at the $k$ th iteration by finding that the palindromic polynomial $p^{(2 k)}$ has no root at +1 . Then $p=(\xi-1)^{2 k} p^{(2 k)}$, which proves that $p$ has a root at +1 of an even multiplicity. It follows by Lemma 3 that an antipalindromic $p$ must have a root at +1 of an odd multiplicity.•
Proof of Lemma 3: The polynomial equation (1) can be written in a matrix-vector form as

$$
\left[\begin{array}{c}
p_{0} \\
p_{1} \\
\vdots \\
p_{n}
\end{array}\right]=\left[\begin{array}{cccc}
-1 & 0 & \cdots & 0 \\
1 & -1 & \ddots & \vdots \\
0 & \ddots & \ddots & 0 \\
\vdots & \ddots & 1 & -1 \\
0 & \cdots & 0 & 1
\end{array}\right]\left[\begin{array}{c}
q_{0} \\
q_{1} \\
\vdots \\
q_{n-1}
\end{array}\right]
$$

where $p_{0}, p_{1}, \ldots, p_{n}$ are the coefficients of $p$ and $q_{0}, q_{1}, \ldots, q_{n-1}$ are the coefficients of $q$. The restriction of the linear mapping $q \mapsto p$, defined by (2), to the subspace of palindromic $q$ is

$$
\left[\begin{array}{c}
p_{0} \\
p_{1} \\
\vdots \\
p_{n}
\end{array}\right]=\left[\begin{array}{cccc}
-1 & 0 & \cdots & 0 \\
1 & -1 & \ddots & \vdots \\
0 & \ddots & \ddots & 0 \\
\vdots & 0 & 1 & -1 \\
\vdots & 0 & -1 & 1 \\
0 & . & . & 0 \\
-1 & 1 & . & \vdots \\
1 & 0 & \cdots & 0
\end{array}\right]\left[\begin{array}{c}
q_{0} \\
q_{1} \\
\vdots \\
q_{m}
\end{array}\right]=\left[\begin{array}{c}
-q_{0} \\
q_{0}-q_{1} \\
\vdots \\
q_{m-1}-q_{m} \\
-q_{m-1}+q_{m} \\
\vdots \\
-q_{0}+q_{1} \\
q_{0}
\end{array}\right] \text {, }
$$

when $n$ is even $(n=: 2 m)$ and

$$
\left[\begin{array}{c}
p_{0} \\
p_{1} \\
\vdots \\
p_{n}
\end{array}\right]=\left[\begin{array}{cccc}
-1 & 0 & \cdots & 0 \\
1 & -1 & \ddots & \vdots \\
0 & \ddots & \ddots & 0 \\
\vdots & \ddots & 1 & -1 \\
\vdots & \ldots & 0 & 0 \\
\vdots & . & -1 & 1 \\
0 & . & . & 0 \\
-1 & 1 & . \cdot & \vdots \\
1 & 0 & \ldots & 0
\end{array}\right]\left[\begin{array}{c}
q_{0} \\
q_{1} \\
\vdots \\
q_{m}
\end{array}\right]=\left[\begin{array}{c}
-q_{0} \\
q_{0}-q_{1} \\
\vdots \\
q_{m-1}-q_{m} \\
0 \\
-q_{m-1}+q_{m} \\
\vdots \\
-q_{0}+q_{1} \\
q_{0}
\end{array}\right] \text {, }
$$

when $n$ is odd $(n=: 2 m+1)$. This shows that the restriction of (2) to the subspace of palindromic arguments, automatically restricts the image to the subspace of antipalindromic vectors $p$. Moreover, there is a one-to-one mapping between $q_{0}, q_{1}, \ldots, q_{m}$ and $p_{0}, p_{1}, \ldots, p_{m}$ given by the equation

$$
\left[\begin{array}{c}
p_{0} \\
p_{1} \\
\vdots \\
p_{m}
\end{array}\right]=\left[\begin{array}{cccc}
-1 & 0 & \cdots & 0 \\
1 & -1 & \ddots & \vdots \\
0 & \ddots & \ddots & 0 \\
0 & 0 & 1 & -1
\end{array}\right]\left[\begin{array}{c}
q_{0} \\
q_{1} \\
\vdots \\
q_{m}
\end{array}\right] .
$$

This proves that given an antipalindromic $p$ there is a unique solution $q$ of (2), which is palindromic. $\bullet$ 
Proof of Lemma 4: Consider (1) with $p$ palindromic The "only if" implication in item 1' of Theorem 2 and the assumption that $p$ is palindromic imply that the roots of $p$ satisfy item 1'. The roots of $q$ are a subset of the roots of $p$ so that they also satisfy item 1'. By the "if" implication of item 1' of Theorem 2, we conclude that $q$ is either palindromic or antipalindromic. Assume that it is palindromic. Then by Lemma $3, p$ is antipalindromic, which is a contradiction. Therefore, $q$ is antipalindromic.

Let $\bar{\lambda}$ be the complex conjugate of $\lambda$. Theorem 2 shows that the complex roots of palindromic and antipalindromic polynomials that are not on the unit circle can be divided into four-tuples $(\lambda, 1 / \lambda, \bar{\lambda}, 1 / \bar{\lambda})$. The complex roots that are on the unit circle and the real roots of $p$, except possibly roots at \pm 1 , can be divided into tuples $(\lambda, 1 / \lambda)$. These properties are common for palindromic and antipalindromic polynomials. The distinguishing property of antipalindromic polynomials is that they have a root at +1 with an odd multiplicity. Conversely, provided that any root $\lambda$, except possibly roots at \pm 1 , has a corresponding root $1 / \lambda$ of the same multiplicity, the polynomial is either palindromic or antipalindromic. The existence of a root at +1 and its multiplicity determine whether the polynomial is palindromic or antipalindromic.

Let $\operatorname{rev}(p)$ denote the "reversed" polynomial of $p$, i.e.,

$$
\operatorname{rev}(p)(\xi):=p_{n} \xi^{0}+p_{n-1} \xi^{1}+\cdots+p_{0} \xi^{n},
$$

where $p(\xi)=p_{0} \xi^{0}+p_{1} \xi^{1}+\cdots+p_{n} \xi^{n}$. Then, obviously $p$ is palindromic/antipalindromic if and only if $p= \pm \operatorname{rev}(p)$ ( + refers to the palindromic case and - refers to the antipalindromic case). Note that the rev operator obeys the following property

$$
\operatorname{rev}(p)(\xi)= \begin{cases}\xi^{n} p(1 / \xi), & \text { for } \xi \neq 0 \\ p_{n}, & \text { for } \xi=0\end{cases}
$$

which gives an algebraic characterisation of palindromic and antipalindromic polynomials, namely $p$ is palindromic/antipalindromic $\Longleftrightarrow$

$$
\left\{\begin{array}{l}
p(\xi) \mp \xi^{n} p(1 / \xi)=0, \quad \text { for all } \xi \in \mathbb{C}, \xi \neq 0, \text { and } \\
p_{0} \mp p_{n}=0 .
\end{array}\right.
$$

Next we state two corollaries of Theorem 2 .

Corollary 5 (Elementary palindromic and antipalindromic polynomials). Any palindromic or antipalindromic polynomial $p$ can be represented as a product of the following five elementary polynomials:

- $e_{1}(\xi):=\xi-1$,

- $e_{2}(\xi):=\xi+1$,

- $e_{3}(\alpha, \xi):=\xi^{2}-\frac{1+\alpha^{2}}{\alpha} \xi+1$, where $\alpha \in \mathbb{R}$,

- $e_{4}(\omega, \xi):=\xi^{2}-2 \cos (\omega) \xi+1$, where $\omega \in \mathbb{R}$, and

- $e_{5}(\lambda, \xi) \quad:=\xi^{4}-2 \Re(\lambda) \frac{1+|\lambda|^{2}}{|\lambda|^{2}} \xi^{3}+$ $\left(2+|\lambda|^{2}+\frac{2 \Re(\lambda)^{2}-\mathfrak{I}(\lambda)^{2}}{|\lambda|^{2}}\right) \xi^{2}-2 \Re(\lambda) \frac{1+|\lambda|^{2}}{|\lambda|^{2}} \xi+1$, where $\lambda \in \mathbb{C}$ is neither purely real nor purely imaginary. i.e., there exist unique scalar $c \in \mathbb{R}$ and parameters $\alpha_{i} \in \mathbb{R}$ for $i=1, \ldots, k_{3}, \omega_{i} \in \mathbb{R}$ for $i=1, \ldots, k_{4}, \lambda_{i} \in \mathbb{C}$ for $i=1, \ldots, k_{5}$, such that

$$
p(\xi)=c e_{1}^{k_{1}}(\xi) e_{2}^{k_{2}}(\xi) \prod_{i=1}^{k_{3}} e_{3}\left(\alpha_{i}, \xi\right) \prod_{i=1}^{k_{4}} e_{4}\left(\omega_{i}, \xi\right) \prod_{i=1}^{k_{5}} e_{5}\left(\lambda_{i}, \xi\right)
$$

$\left(k_{i}=0\right.$ means that there is no elementary polynomial of the ith type). If $p$ is palindromic $k_{1}$ is even and if $p$ antipalindromic $k_{1}$ is odd. Conversely, any product (6) of elementary polynomials is palindromic if $k_{1}$ is even and antipalindromic if $k_{1}$ is odd.

Proof: Observe that

- the root of $e_{1}(\xi)$ is +1 ,

- the root of $e_{2}(\xi)$ is -1 ,

- the roots of $e_{3}(\alpha, \xi)$ are $\alpha$ and $1 / \alpha$,

- the roots of $e_{4}(\omega, \xi)$ are $e^{\mathbf{i} \omega}$ and $e^{-\mathbf{i} \omega}$, and

- the roots of $e_{5}(\lambda, \xi)$ are $\lambda, 1 / \lambda, \bar{\lambda}$, and $1 / \bar{\lambda}$,

so that the factorisation (6) corresponds to the grouping of the roots of $p$ into roots at \pm 1 , tuples on the real axis, tuples on the unit circle, and four-tuples in the complex plane, as done in the proof of Theorem 2. Therefore, Corollary 5 is a restatement of Theorem 2 in terms of factor polynomials instead of groups of roots.

Corollary 6 (Algebraic properties of the palindromic and antipalindromic polynomials). The following hold:

- "palindromic" $\times$ "palindromic" is palindromic,

- "palindromic" $\times$ "antipalindromic" is antipalindromic,

- "antipalindromic" $\times$ "antipalindromic" is palindromic.

\section{PALINDROMIC/ANTIPALINDROMIC POLYNOMIALS IN SYSTEM THEORY}

In the remaining part of the paper, we study the relevance of palindromic and antipalindromic polynomials in system theory. In Section III-A, we show that difference equation representations of LTI time-reversible systems are related to palindromic/antipalindromic polynomials. In Section III-B, we show that palindromic/antipalindromic polynomials also have a link with representations of LTI systems that have special quadratic functionals associated with them, known as conserved quantities, i.e., functionals that remain constant along the trajectories of the system. We begin with a short description of LTI systems and quadratic difference forms.

Define by $\sigma$ the shift operator

$$
(\sigma w)(t):=w(t+1) .
$$

An LTI system can always be represented in, what is called kernel form, as

$$
\mathscr{B}=\operatorname{ker}(R(\sigma)):=\left\{w \in\left(\mathbb{R}^{\mathrm{w}}\right)^{\mathbb{Z}} \quad \mid \quad R(\sigma) w=0\right\},
$$

where $R \in \mathbb{R}^{\bullet \times \mathrm{w}}[\sigma]$. Note that $\mathbb{R}^{\bullet \times \mathrm{w}}[\sigma]$ denotes the space of all polynomial matrices with $w$ columns and an unspecified number of rows. The representation (7) is called minimal if 
$R$ has the minimum number of rows among all the kernel representations of $\mathscr{B}$.

A quadratic difference form [Kan] is the discrete-time analogue of quadratic differential form for continuous-time systems introduced in [WT98]. Let $\Phi \in \mathbb{R}^{\mathrm{w} \times \mathrm{w}}[\zeta, \eta]$, i.e., $\Phi(\zeta, \eta)=\sum_{i, j=0}^{n} \Phi_{i, j} \zeta^{i} \eta^{j}$, where $\Phi_{i, j} \in \mathbb{R}^{\mathrm{w} \times \mathrm{w}}$ for all $i, j \in$ $\{0,1, \ldots, n\}$ and $n$ is a natural number. Such a polynomial $\Phi$ induces a quadratic difference form $(\mathrm{QDF})$ on $\left(\mathbb{R}^{\mathrm{w}}\right)^{\mathbb{Z}}$ as

$Q_{\Phi}:\left(\mathbb{R}^{\mathrm{w}}\right)^{\mathbb{Z}} \mapsto \mathbb{R}^{\mathbb{Z}}, \quad Q_{\Phi}(w)(t):=\sum_{i, j=0}^{n} w(t+i)^{\top} \Phi_{i, j} w(t+j)$.

We call $\Phi$ symmetric if $\Phi(\zeta, \eta)=\Phi(\eta, \zeta)^{\top}$. In this paper, we consider only the set of symmetric QDFs, denoted by $\mathbb{R}_{s}^{\mathrm{W} \times \mathrm{w}}[\zeta, \eta]$, because every nonsymmetric QDF is equivalent to a symmetric one.

We describe the notion of the rate of change of a QDF, which will be used to obtain results about conserved quantities later on in the paper. The rate of change $\nabla Q_{\Phi}$ of a QDF $Q_{\Phi}$ is defined as

$$
\nabla Q_{\Phi}(w)(t):=Q_{\Phi}(w)(t+1)-Q_{\Phi}(w)(t) .
$$

Let $\Psi(\zeta, \eta)$ be the two-variable polynomial matrix associated with the QDF $\nabla Q_{\Phi}$. Then, it is easy to see that

$$
\Psi(\zeta, \eta)=(\zeta \eta-1) \Phi(\zeta, \eta)
$$

A QDF $Q_{\Phi}$ is said to be zero along a behaviour $\mathscr{B}$ (denoted by $\left.Q_{\Phi} \stackrel{\mathscr{B}}{=} 0\right)$ if $Q_{\Phi}(w)(t)=0$, for all $w \in \mathscr{B}$ and $t \in \mathbb{Z}$. The next proposition which is an analogue of Proposition 3.2 of [WT98] gives the condition on a two-variable polynomial under which the QDF associated with it is zero along a given scalar autonomous behaviour.

Proposition 7. Let $\Phi \in \mathbb{R}_{S}[\zeta, \eta]$ and let $\mathscr{B}=\operatorname{ker}(r(\sigma))$, where $r \in \mathbb{R}[\sigma]$. Then $Q_{\Phi} \stackrel{\mathscr{B}}{=} 0$ if and only if there exists $f \in \mathbb{R}[\zeta, \eta]$, such that

$$
\Phi(\zeta, \eta)=f(\eta, \zeta) r(\eta)+r(\zeta) f(\zeta, \eta)
$$

Proof: The proof can be deduced from Lemma A.1, p.1734, [WT98].

Equip the set of QDFs associated with a behaviour $\mathscr{B}$ with the equivalence relation defined by

$$
Q_{\Phi} \stackrel{\mathscr{B}}{\sim} Q_{\Psi} \quad \Longleftrightarrow \quad Q_{\Phi}(w)=Q_{\Psi}(w), \quad \text { for all } w \in \mathscr{B} .
$$

It is easy to see that the set of equivalence classes under $\stackrel{\mathscr{B}}{\sim}$ is a linear vector space over $\mathbb{R}$. With every equivalence class of QDFs associated with an autonomous behaviour $\mathscr{B}$, we associate a certain representative known as the $\mathscr{B}$-canonical representative. Below, we define the notion of $\mathscr{B}$-canonicity of QDFs.

Definition 8. Let $\mathscr{B}$ be an autonomous behaviour given by $\mathscr{B}=\operatorname{ker}(r(\sigma))$, where $r \in \mathbb{R}[\sigma]$. Then a QDF $Q_{\Phi}$ is $\mathscr{B}$ canonical if $r(\zeta)^{-1} \Phi(\zeta, \eta) r(\eta)^{-1}$ is strictly proper.

If $r$ has degree $n$, then from the definition, it follows that the two-variable polynomials associated with $\mathscr{B}$-canonical QDFs are spanned by monomials $\zeta^{i} \eta^{j}$, with $i, j \leq n-1$. It is easy to see that every QDF has a $\mathscr{B}$-canonical representative.

\section{A. Time-reversible systems}

Next, we study kernel representations of discrete-time time-reversible scalar autonomous and SISO systems. Here, we show that there exists an inherent link between palindromic/antipalindromic polynomials and the kernel representation of time-reversible systems.

In Section II, we defined the reversed polynomial $\operatorname{rev}(p)$ (see (3)). By viewing the polynomial $p$ as the vector $\operatorname{col}\left(p_{0}, p_{1}, \ldots, p_{n}\right)$ of its coefficients, we can naturally extend the "rev" operator for vectors. Thus

$$
\operatorname{rev}\left(\operatorname{col}\left(p_{0}, p_{1}, \ldots, p_{n}\right)\right):=\operatorname{col}\left(p_{n}, p_{n-1}, \ldots, p_{0}\right) .
$$

We define the "rev" operator for a sequence $w \in\left(\mathbb{R}^{w}\right)^{\mathbb{Z}}$ as

$$
(\operatorname{rev}(w))(t):=w(-t), \quad \text { for all } t \in \mathbb{Z} .
$$

Thus the "rev" operator acting on a sequence reverses the order of the sequence. It is easy to see that $\operatorname{rev}(\operatorname{rev}(w))=w$.

Definition 9 (Time-reversible system). A dynamical system $\mathscr{B}$ is time-reversible if $w \in \mathscr{B}$ implies $\operatorname{rev}(w) \in \mathscr{B}$.

We now give a necessary and sufficient condition in terms of a kernel representation for an autonomous scalar system to be time-reversible.

Theorem 10. The LTI system $\mathscr{B}=\operatorname{ker}(p(\sigma)), p \in \mathbb{R}[\xi]$, is time-reversible if and only if $p$ is either palindromic or antipalindromic.

Proof: $\quad(\Longrightarrow)$ Let $\mathscr{B}=\operatorname{ker}(p(\sigma))$ and $p(\sigma)=$ $\sum_{i=0}^{n} p_{i} \sigma^{i}$. Assume that $\operatorname{rev}(w) \in \mathscr{B}$. Then

$(p(\sigma) \operatorname{rev}(w))(t)=\sum_{i=0}^{n}\left(p_{i} \sigma^{i} \operatorname{rev}(w)\right)(t)=\sum_{i=0}^{n} p_{i} w(-t-i)=0$,

for every $t \in \mathbb{Z}$. Putting $i=n-j$ and $t=-t_{1}-n$ in the above equation, we get

$$
\sum_{j=0}^{n} p_{n-j} w\left(t_{1}+j\right)=0, \quad \text { for every } t_{1} \in \mathbb{Z} .
$$

Since $\mathscr{B}$ is time-reversible, $w \in \mathscr{B}$, i.e.,

$$
\sum_{j=0}^{n} p_{j} w\left(t_{1}+j\right)=0, \quad \text { for every } t_{1} \in \mathbb{Z} .
$$

From equations (8) and (9),

$$
\sum_{j=0}^{n} p_{n-j} w\left(t_{1}+j\right)=k \sum_{j=0}^{n} p_{j} w\left(t_{1}+j\right), \quad \text { for every } t_{1} \in \mathbb{Z} \text {. }
$$

where $k \in \mathbb{R}$. Since $\mathscr{B}$ is linear, it follows that $k p=\operatorname{rev}(p)$, where $p=\operatorname{col}\left(p_{0}, p_{1}, \ldots, p_{n}\right)$. Therefore, $k^{2}=1$ or $k= \pm 1$ and hence $p(\sigma)$ is either palindromic or antipalindromic.

$(\Longleftarrow) \quad$ Assume that $p(\sigma)=\sum_{i=0}^{n} p_{i} \sigma^{i}$ is palindromic/antipalindromic. Then $p_{i}= \pm p_{n-i}$ for $i=0,1, \ldots, n$. Let $\mathscr{B}=\operatorname{ker}(p(\sigma))$. For any $w \in \mathscr{B}$, we have $\sum_{i=0}^{n} p_{i} \sigma_{i} w=0$. The left hand side can be written as

$$
\sum_{i=0}^{n} p_{i} w(t+i)= \pm \sum_{i=0}^{n} p_{n-i} w(t+i)
$$


Putting $i=n-j$ and $t=-t_{1}-n$, we get

$$
\pm \sum_{j=0}^{n} p_{j} w\left(-t_{1}-j\right)= \pm \sum_{j=0}^{n}\left(p_{j} \sigma^{j} \operatorname{rev}(w)\right)\left(t_{1}\right)=0,
$$

for every $t_{1} \in \mathbb{Z}$. Thus if $p$ is palindromic/antipalindromic, $w \in \mathscr{B}$ implies $\operatorname{rev}(w) \in \mathscr{B}$, or $\mathscr{B}$ is reversible.

Next we give a condition in terms of a kernel representation for a SISO system to be time-reversible.

Theorem 11. Let $\mathscr{B}=\operatorname{ker}([q(\sigma)-p(\sigma)])$, where $p \in$ $\mathbb{R}[\sigma]$ is of degree $\mathrm{p}$ and $q \in \mathbb{R}[\sigma]$ is of degree $\mathrm{q} \leq \mathrm{p}$. Define $\mathrm{d}:=\mathrm{p}-\mathrm{q}$. The system $\mathscr{B}$ is time-reversible if and only if i) $q$ has d roots at zero and ii) $p$ and $q^{\prime}(\sigma):=q(\sigma) / \sigma^{\mathrm{d}}$ are either both palindromic or both antipalindromic. If $\mathscr{B}$ is controllable, then both $p$ and $q^{\prime}$ are palindromic.

Proof: Let $p(\sigma)=\sum_{i=0}^{\mathrm{p}} p_{i} \sigma^{i}$ and $q(\sigma)=\sum_{i=0}^{\mathrm{q}} q_{i} \sigma^{i}$. For any $\operatorname{col}(y, u) \in \mathscr{B}:=\operatorname{ker}([p(\sigma)-q(\sigma)])$, we have

$$
\sum_{i=0}^{\mathrm{p}} p_{i} y(t+i)=\sum_{i=0}^{\mathrm{q}} q_{i} u(t+i), \quad \text { for every } t \in \mathbb{Z} .
$$

(If) Assume that $q$ is divisible by $\sigma^{\mathrm{d}}$ and that $p$ and $q_{1}$ are palindromic, where $q_{1}(\sigma):=q(\sigma) / \sigma^{d}$. Then $p_{i}=p_{n-i}$ for $i=0,1, \ldots, \mathrm{p}, q_{i}=0$ for $i=0, \ldots, \mathrm{d}-1$, and $q_{i}=q_{\mathrm{p}-i}$ for $i=\mathrm{d}, \ldots, \mathrm{q}$. From (10),

$$
\sum_{i=0}^{\mathrm{p}} p_{\mathrm{p}-i} y(t+i)=\sum_{i=\mathrm{d}}^{\mathrm{q}} q_{\mathrm{p}-i} u(t+i), \quad \text { for every } t \in \mathbb{Z} .
$$

Putting $i=\mathrm{p}-j$, and $t=-t_{1}-\mathrm{p}$ in the above equation, we get

$$
\sum_{j=0}^{\mathrm{p}} p_{j} y\left(-t_{1}-j\right)=\sum_{j=\mathrm{d}}^{\mathrm{q}} q_{j} u\left(-t_{1}-j\right), \quad \text { for every } t_{1} \in \mathbb{Z}
$$

or

$$
\sum_{j=0}^{\mathrm{p}}\left(p_{j} \sigma^{j} \operatorname{rev}(y)\right)=\sum_{j=\mathrm{d}}^{\mathrm{q}}\left(q_{j} \sigma^{j} \operatorname{rev}(u)\right)
$$

so that

$$
[p(\sigma)-q(\sigma)] \operatorname{rev}(\operatorname{col}(y, u))=0 .
$$

This implies that $\operatorname{rev}(\operatorname{col}(y, u)) \in \mathscr{B}$. Hence $\mathscr{B}$ is reversible. The proof for the case when $p$ and $q$ are both antipalindromic is similar.

(Only If) Assume that $\mathscr{B}$ is time-reversible and $\operatorname{rev}(\operatorname{col}(y, u)) \in \mathscr{B}$. We have

$$
\begin{gathered}
\sum_{i=0}^{\mathrm{p}} p_{i} \sigma^{i} \operatorname{rev}(y)=\sum_{i=0}^{\mathrm{q}} q_{i} \sigma^{i} \operatorname{rev}(u) \\
\Longrightarrow \sum_{i=0}^{\mathrm{p}} p_{i} y(-t-i)=\sum_{i=0}^{\mathrm{q}} q_{i} u(-t-i), \quad \text { for every } t \in \mathbb{Z} .
\end{gathered}
$$

Putting $i=\mathrm{p}-j$ and $t=-t_{1}-\mathrm{p}$ in the above equation, we get

$$
\sum_{j=0}^{\mathrm{p}} p_{\mathrm{p}-j} y\left(t_{1}+j\right)-\sum_{j=\mathrm{d}}^{\mathrm{p}} q_{\mathrm{p}-j} u\left(t_{1}+j\right)=0, \quad \text { for every } t_{1} \in \mathbb{Z}
$$

Let

$$
\begin{array}{r}
W\left(t_{1}\right):=\operatorname{col}\left(y\left(t_{1}\right), y\left(t_{1}+1\right), \ldots, y\left(t_{1}+p\right), u\left(t_{1}\right), \ldots,\right. \\
\left.u\left(t_{1}+\mathrm{d}-1\right), u\left(t_{1}+\mathrm{d}\right), u\left(t_{1}+\mathrm{d}+1\right), \ldots, u\left(t_{1}+\mathrm{p}\right)\right)
\end{array}
$$

then

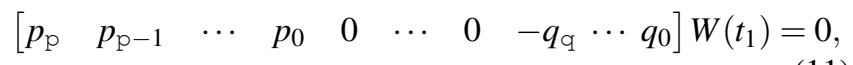

for every $t_{1} \in \mathbb{Z}$. Since $\mathscr{B}$ is time-reversible, $\operatorname{col}(y, u) \in \mathscr{B}$, i.e.,

$$
\sum_{j=0}^{\mathrm{p}} p_{j} y\left(t_{1}+j\right)-\sum_{j=0}^{\mathrm{q}} q_{j} u\left(t_{1}+j\right)=0, \quad \text { for every } t_{1} \in \mathbb{Z}
$$

or

$$
\left[\begin{array}{llllllllll}
p_{0} & p_{1} & \cdots & p_{\mathrm{p}} & -q_{0} & \cdots & -q_{\mathrm{q}} & 0 & \cdots & 0
\end{array}\right] W\left(t_{1}\right)=0,
$$

for every $t_{1} \in \mathbb{Z}$. Since $\mathscr{B}$ is linear, from equations (11) and (12), it follows that

$$
\begin{aligned}
& {\left[\begin{array}{llllllllll}
p_{\mathrm{p}} & p_{\mathrm{p}-1} & \cdots & p_{0} & 0 & \cdots & 0 & -q_{\mathrm{q}} & \cdots & q_{0}
\end{array}\right]} \\
& =k\left[\begin{array}{llllllllll}
p_{0} & p_{1} & \cdots & p_{\mathrm{p}} & -q_{0} & \cdots & -q_{\mathrm{q}} & 0 & \cdots & 0
\end{array}\right]
\end{aligned}
$$

where $k \in \mathbb{R}$. It is easy to see that $k= \pm 1$. When $k=1, p$ is palindromic, $q_{i}=0$ for $i=0, \ldots, \mathrm{d}-1$, and $q_{i}=q_{\mathrm{p}-i}$ for $i=$ $\mathrm{d}, \ldots, \mathrm{q}$, i.e., $q(\sigma)$ is divisible by $\sigma^{d}$ and $q^{\prime}(\sigma)=q(\sigma) / \sigma^{d}$ is palindromic. When $k=-1, p$ is antipalindromic, $q_{i}=0$ for $i=0, \ldots, \mathrm{d}-1$, and $q_{i}=-q_{\mathrm{p}-i}$ for $i=\mathrm{d}, \ldots, \mathrm{q}$, i.e., $q(\sigma)$ is divisible by $\sigma^{d}$ and $q^{\prime}(\sigma)=q(\sigma) / \sigma^{d}$ is antipalindromic.

If in addition, $\mathscr{B}$ is controllable, then $p$ and $q$ are co-prime. If both $p$ and $q^{\prime}$ are antipalindromic, then from Lemma 3, both $p$ and $q^{\prime}$ have at least one root at 1 . Since $q$ is divisible by $q^{\prime}$, this implies that $p$ and $q$ are not co-prime which is a contradiction. Hence if $\mathscr{B}$ is controllable and time-reversible, then $p$ and $q^{\prime}$ are both palindromic.

Using the steps followed in the proof of the above theorem, one can also deduce the structure of the kernel representation of a multi-input single output (MISO) time-reversible system.

\section{B. Existence of conserved quantities}

The notion of a conserved quantity is defined in [RW05] for the case of continuous-time systems. Below, we give an analogous definition for the case of discrete-time systems.

Definition 12. Let $\mathscr{B}$ be a linear autonomous behaviour. $A$ $Q D F Q_{\Phi}$ is a conserved quantity for $\mathscr{B}$ if

$$
\nabla Q_{\Phi}(w)=0, \quad \text { for all } w \in \mathscr{B} .
$$

In other words, a conserved quantity for a behaviour $\mathscr{B}$ is a QDF, which remains constant upon acting on any sequence belonging to the behaviour. In this section, we examine the conditions on the representation of a scalar autonomous behaviour under which it has a conserved quantity associated with it. We begin with the following definition.

Definition 13. The maximal palindromic factor of $r \in \mathbb{R}[\xi]$ is its monic palindromic factor of maximal degree. 
For any given polynomial $r \in \mathbb{R}[\xi]$, it is easy to see that there exists a unique maximal palindromic factor. In the next theorem, we examine the conditions under which a linear behaviour $\mathscr{B}$ has conserved quantities associated with it.

Theorem 14. Consider a linear behaviour $\mathscr{B}=\operatorname{ker}(r(\sigma))$, where $r \in \mathbb{R}[\sigma]$ has no root at zero. There exists a nonzero conserved quantity for $\mathscr{B}$ if and only if either $r$ has a nonunity maximal palindromic factor $r^{\prime}$ or $r(\sigma)=\sigma-1$. Moreover if $v:=r / r^{\prime}$ is such that $v(1) \neq 0$, then the dimension of the space of conserved quantities is $\left\lfloor\left(\operatorname{deg}\left(r^{\prime}\right)-1\right) / 2\right\rfloor$, otherwise it is $\left\lfloor\left(\operatorname{deg}\left(r^{\prime}\right)+1\right) / 2\right\rfloor$, where $\lfloor m\rfloor$ is the greatest integer less than or equal to $m$.

Proof: Let the degree of $r$ be equal to $n$. Let $r=r^{\prime} q$, where $r^{\prime}$ the maximal palindromic factor of $r$, and let $n^{\prime}$ denote the degree of $r^{\prime}$. Assume that $\mathscr{B}$ has a conserved quantity whose two-variable polynomial representation is $\phi(\zeta, \eta)$. Then

$$
\phi(\zeta, \eta)=\frac{r(\zeta) f^{\prime}(\zeta, \eta)+r(\eta) f^{\prime}(\eta, \zeta)}{\zeta \eta-1},
$$

for some $f^{\prime} \in \mathbb{R}[\zeta, \eta]$. It is easy to see that since $\phi$ is $\mathscr{B}$ canonical, $f^{\prime}$ is independent of $\zeta$ and is of degree less than or equal to $n-1$ in $\eta$. Hence

$$
\phi(\zeta, \eta)=\frac{r(\zeta) f(\eta)+r(\eta) f(\zeta)}{\zeta \eta-1}
$$

where $f(\eta)=f^{\prime}(\zeta, \eta)$. Since $\phi$ exists, the numerator is divisible by $\zeta \eta-1$. Consequently by factor theorem,

$$
\xi^{n}\left(r(\xi) f\left(\xi^{-1}\right)+r\left(\xi^{-1}\right) f(\xi)\right)=0 .
$$

This implies that

$$
\xi^{n} f\left(\xi^{-1}\right) q(\xi)=-\xi^{n-n^{\prime}} q\left(\xi^{-1}\right) f(\xi) .
$$

Define $s(\xi):=\xi^{n-n^{\prime}} f(\xi) q\left(\xi^{-1}\right)$ and observe that

$$
s(\xi)+\xi^{2 n-n^{\prime}} s\left(\xi^{-1}\right)=0 .
$$

Two cases arise.

Case 1: $v$ does not have a root at 1 . In this case, for equation (15) to hold, it is easy to see that $f$ should be of the form $f(\xi)=v(\xi) f_{a}(\xi)$, where $f_{a}$ is an antipalindromic polynomial such that

$$
\xi^{n^{\prime}} f_{a}\left(\xi^{-1}\right)+f_{a}(\xi)=0 .
$$

Since the degree of $f$ is less than $n$, the degree of $f_{a}$ is less than $n^{\prime}$. This implies that $f_{a}$ has at least one root at zero. If $n^{\prime}$ is even, then the general expression for $f_{a}$ can be written as

$$
f_{a}(\xi)=\sum_{i=1}^{n^{\prime} / 2-1} p_{i}\left(\xi^{i}-\xi^{n^{\prime}-i}\right)
$$

whereby the dimension of the space of all possible polynomials $f_{a}$ is $n^{\prime} / 2-1$. If $n^{\prime}$ is odd, then the general expression for $f_{a}$ can be written as

$$
f_{a}(\xi)=\sum_{i=1}^{\frac{n^{\prime}-1}{2}} p_{i}\left(\xi^{i}-\xi^{n^{\prime}-i}\right)
$$

whereby the dimension of the space of all possible polynomials $f_{a}$ is $\left(n^{\prime}-1\right) / 2$. From (14), it can be seen that there is a one-one correspondence between $\phi$ and any $f$ of degree less than or equal to $n-1$. Hence the dimension of conserved quantities for the two subcases of $n^{\prime}$ being even and odd is the same as the dimension of all possible polynomials $f_{a}$ for the respective subcases, which is equal to $\left\lfloor\left(n^{\prime}-1\right) / 2\right\rfloor$.

Case 2: $v$ has a root at 1 . Let $v(\xi)=(\xi-1) v^{\prime}(\xi)$. In this case, for (15) to hold, it is easy to see that $f$ should be of the form $f(\xi)=v^{\prime}(\xi) f^{\prime}(\xi)$, where $f^{\prime}$ is a palindromic polynomial, such that

$$
\xi^{n^{\prime}+1} f^{\prime}\left(\xi^{-1}\right)-f^{\prime}(\xi)=0
$$

Since the degree of $f$ is less than $n$, the degree of $f^{\prime}$ is less than or equal to $n^{\prime}$. This implies that $f^{\prime}$ has at least one root at zero. If $n^{\prime}$ is even, the general expression for $f^{\prime}$ can be written as

$$
f^{\prime}(\xi)=\sum_{i=1}^{n^{\prime} / 2} p_{i}\left(\xi^{i}+\xi^{n^{\prime}+1-i}\right)
$$

whereby the dimension of the space of all possible polynomials $f^{\prime}$ is $n^{\prime} / 2$. If $n^{\prime}$ is odd, the general expression for $f^{\prime}$ can be written as

$$
f^{\prime}(\xi)=\sum_{i=1}^{\frac{n^{\prime}-1}{2}} p_{i}\left(\xi^{i}+\xi^{n^{\prime}+1-i}\right)+p_{\frac{n_{i}+1}{2}} \xi^{\frac{n_{i}+1}{2}}
$$

whereby the dimension of the space of all possible polynomials $f^{\prime}$ is $\left(n^{\prime}+1\right) / 2$. From (14), it can be seen that there is a one-one correspondence between $\phi$ and any $f$ of degree less than or equal to $n-1$. Hence the dimension of conserved quantities for the two subcases of $n^{\prime}$ being even and odd is the same as the dimension of all possible polynomials $f^{\prime}$ for the respective subcases, which is equal to $\left\lfloor\left(n^{\prime}+1\right) / 2\right\rfloor$.

From the above theorem and from the discussion on timereversible systems, it can be inferred that every reversible scalar system has conserved quantities associated with it.

\section{REFERENCES}

[FW91] F. Fagnani and J. C. Willems. Representations of time-reversible systems. J. of Math. Systems, Estimation and Control, 1:5-28, 1991.

[Kan] O. Kaneko. Studies on discrete time dissipative systems in a behavioral framework $\mathrm{PhD}$ thesis, Graduate School of Engineering Science, Osaka University, October 2005

[PW97] J.W. Polderman and J.C. Willems, Introduction to Mathematical System theory: A Behavioral Approach Springer-Verlag, Berlin, 1997.

[RW05] P. Rapisarda and J. C. Willems. Conserved- and zero-mean quadratic quantities in oscillatory systems. Math. Contr. Sign. Syst., 17:173-200, 2005.

[WT98] J. C. Willems and H. L. Trentelman. On quadratic differential forms. SIAM Journal on Control and Optimization, 36:1702-1749, 1998. 